\title{
Civilisations
}

Revue internationale d'anthropologie et de sciences

humaines

61-2 | 2013

Chamanismes en mouvement

\section{Entre « chamanisme » et politique}

Les transmigrations du discours autochtone à la recherche du tempo de la résistance

\section{Nadège Mazars}

\section{OpenEdition}

\section{Journals}

Édition électronique

URL : http://journals.openedition.org/civilisations/3264

DOI : 10.4000/civilisations.3264

ISSN : 2032-0442

\section{Éditeur}

Institut de sociologie de l'Université Libre de Bruxelles

\section{Édition imprimée}

Date de publication : 28 juin 2013

Pagination : 37-52

ISSN : 0009-8140

Référence électronique

Nadège Mazars, «Entre «chamanisme » et politique », Civilisations [En ligne], 61-2 | 2013, mis en ligne le 14 juin 2013, consulté le 01 mai 2019. URL : http://journals.openedition.org/civilisations/3264 ;

DOI : 10.4000/civilisations.3264 


\title{
Entre «chamanisme " et politique \\ Les transmigrations du discours autochtone à la recherche \\ du tempo de la résistance
}

\author{
Nadège MAZARS
}

\begin{abstract}
Résumé : En retraçant l'histoire d'Edinson A., cet article s'interroge sur les relations entre « chamanisme 》 et politique, et sur les conditions sociales d'émergence d'un discours appuyant la construction d'une autonomie territoriale et politique des communautés autochtones dans le Cauca colombien. Éduqué par les religieux afin qu'il oublie ses origines, puis s'engageant dans les récupérations de terres des années 1980, il devient combattant de la guérilla autochtone Quintín Lame pour revenir dans les années 1990 à l'apprentissage du rôle social qu'on lui avait prédit dès ses treize ans, à savoir thê'wala, ou figure du "chamane » chez le peuple nasa. Sa trajectoire permet d'illustrer l'articulation de luttes pour la redistribution et pour la reconnaissance, bases des mobilisations autochtones, et les processus d'ethnogenèse qui appuient la pratique et l'action politique. En effet, Edinson est aussi l'un des promoteurs de la « médecine traditionnelle » dans le cadre d'un organisme d'affiliation à la sécurité sociale géré par l'organisation autochtone départementale, le CRIC. En défendant une pratique autre de la santé, l'organisation cherche à obtenir des ressources supplémentaires et à faire reconnaître sa capacité à gouverner. Dans un même mouvement, elle légitime son intervention auprès des communautés en contribuant à construire leur autonomie.
\end{abstract}

Mots-clés : Colombie, santé, mouvement indigène, discours et pratique, autonomie territoriale et politique.

\begin{abstract}
By retracing the biography of Edinson A., this article questions the relation between « shamanism » and politics, and unpacks the conditions of emergence of a discourse supporting the territorial and political autonomy of the indigenous communities in the Colombian Cauca. Edinson's Christian education induced him to forget his origins. He later engaged in the land recuperation movements in the 1980s, and eventually became a fighter in the Quintin Lame indigenous guerrilla. In the 1990s he turned to apprenticeship of the thê'wala, or "shaman", social function that was predicted to him thirteen years earlier among the Nasa people. Edinson's trajectory illustrates the articulation between the indigenous mobilisations for redistribution and for acknowledgement, and the process of ethnogenesis that supports political action and practice. Edinson is presently a promoter of "traditional medicine" in a social security organism managed by a departmental indigenous organization, the CRIC. By defending a different health practice, the organisation seeks to obtain additional resources and to demonstrate its capacity to govern. Furthermore, it legitimises its own intervention within the communities by contributing to their autonomy.
\end{abstract}

Keywords: Columbia, health, indigenous movement, discourse and practice, territorial and political autonomy. 
$\mathrm{J}_{\mathrm{d}+\mathrm{s}}$ 'ai rencontré Edinson A. ${ }^{1}$ pour la première fois en août 2008. Dans le cadre de mon doctorat, je cherchais alors à interviewer l'un des promoteurs de la « médecine traditionnelle » dans les communautés du Cauca colombien. Après deux heures de discussion sur ses connaissances de thê 'wala, rôle social qui se rapproche le plus de la catégorie générique de « chamane » chez le peuple nasa, et alors que je m'apprêtais à clore l'entrevue, Edinson évoqua son parcours au sein de la guérilla autochtone du Quintín Lame, estimant sans aucun doute que je passais à côté d'une des étapes décisives de sa vie. Ce que j'analysais au sortir de l'entretien comme une parenthèse, certes captivante mais indépendante de son parcours de thếwala, m'apparut avec le recul comme l'une des clefs de compréhension de son engagement dans cette voie. La trajectoire d'Edinson n'a rien de linéaire. En cela, elle illustre celle des communautés autochtones, les racines de leur soumission à l'ordre colonial et à son héritier, l'ordre républicain, les conditions de leur émancipation et leur capacité à générer une parole les représentant.

Plus généralement, cet article propose de revenir sur le rôle du discours et de la pratique chamanique dans la consolidation sociale et historique d'un " contre-public », c'est-à-dire d'un espace public alternatif au dominant (Fraser 2011 : 126-128). Système d'interprétation symbolique contraint par un environnement pratique (Perrin 1997: 92), le chamanisme possède aussi une dimension proprement politique qui conduit ses agents à intervenir concrètement dans l'espace social et à mobiliser autant une logique symbolique que matérielle. On propose donc ici d'analyser, à partir de la trajectoire d'Edinson, l'usage du chamanisme dans la construction du discours autochtone et dans la consolidation d'une autonomie territoriale et politique des communautés. Le discours autochtone n'est pas figé. Confondu avec le discours ethnique, il reflète une lutte pour la reconnaissance. Mais ce n'est là qu'une de ces dimensions. Le discours autochtone exprime aussi une demande d'égalité sociale et de redistribution.

\section{Apprendre que l'on est choisi pour devenir thê'wala}

Quand Edinson ressent pour la première fois les « signes », il n'a que treize ans. Il évoque le moment de façon très détaillée. Nous sommes en 1977. Son père et deux de ses oncles se réchauffent auprès du feu dans la maison familiale située en haut de la cordillère, à deux heures de marche de la première route. Ils discutent en buvant de la chicha, une boisson fermentée à base de maïs. L'un de ses oncles est thê'wala, un " grand sage " dans la langue nasa yuwe, qui connaît les plantes, interprète et soigne les maladies. Ils discutent à ce moment de « signes » qu'ils ressentent simultanément et qu'ils expliquent comme annonçant l'arrivée prochaine de visiteurs. Edinson écoute attentivement, il est assis sur le sol, au milieu des « anciens ». Il ressent alors le « coup de courant» dans sa jambe droite, comme si quelqu'un venait de le frapper. Il le dit immédiatement à ses parents qui le regardent, surpris et pensent un instant

1. L'anonymat est ici conservé. Deux entretiens ont été réalisés, l'un en août 2008, et le second en décembre 2011. Dans les deux cas, la rencontre a eu lieu sur le devant de sa maison située en zone rurale de la municipalité d'Inzá, non loin du parc archéologique de Tierradentro. 
qu'il plaisante. Mais l'oncle thê'wala confirme la manifestation de ce qui est interprété comme la révélation d'un pouvoir: il a été choisi pour devenir thê'wala ${ }^{2}$.

Les « signes », ces pulsations ou vibrations ressenties en certains endroits du corps, sont au cœur de la connaissance du thê'wala. Ils indiquent un événement, une menace, un possible déséquilibre (Portela Guarín 2002:106-107) dans les " relations sociocosmiques » (Drexler 2007:149). Car la cosmovision nasa repose sur une conception écocosmologique (Drexler 2007:147) qui ne sépare pas le monde des humains de celui des non-humains (la nature, le domaine de l'invisible) et qui conçoit l'existence de rapports " sociaux » entre chacune des composantes de cet univers. Par les signes qu'il ressent, pouvoir qu'un esprit associé à la nature lui a octroyé, le thê'wala est à même d'interpréter l'état de ces relations et d'identifier les rééquilibrages nécessaires. Il occupe donc dans la société nasa une place spécifique impliquant une responsabilité puisqu'il est une sorte de médiateur qui, par ses actions dans un domaine inaccessible au commun, influe sur le devenir de la communauté.

D'ailleurs, Edinson se rappelle avoir ressenti beaucoup de joie et de fierté au moment où il apprend qu'il possède ce pouvoir. Il pourra donc suivre l'exemple familial et occuper une position sociale spécifique. «J'arriverai à être comme eux !», se rappellet-il avoir pensé. Pourtant, ce n'est que très tardivement, après 1994, qu'il revient à son apprentissage de thê'wala. L'épisode, bien que significatif car il marque un devenir possible autour duquel se rattache tout un univers d'interprétation symbolique, n'est à ce stade pas suffisant pour déterminer sa trajectoire. En dépit d'un environnement familial propice, car d'autres oncles sont thê'wala, il suit la trajectoire de son père, qui lui aussi ressent les « signes » mais n'a jamais appris à les interpréter, ni à manier les plantes. De plus, l'oncle qui lui a confirmé son pouvoir meurt quelques années après et le jeune garçon perd progressivement l'intérêt pour l'apprentissage, tout en continuant à ressentir les signes. Le thê'wala de la communauté de Tumbichucué l'encourage un temps, en lui indiquant les plantes qu'il doit semer. Mais il ne suit pas les conseils et en vient même à éprouver un rejet pour la pratique du mâchage qu'implique la fonction:

\begin{abstract}
Quand j'étais jeune, j'étais récalcitrant au mâchage de coca. Parce qu'ici nous méditons en mâchant de la coca. Nous la grillons... le mambear ${ }^{3}$ c'est... de la coca et du mambe. Ça me donnait du dégoût de voir un vieux, là, mâchant, et ce mâchage de coca dégoulinant comme ça... Je disais, "je vais faire la même chose... Pour faire quoi?" Et pour cette raison ... eh bien... Et en même temps, oui... je voulais être médecin, connaître les plantes, mais à la fois ne pas mâcher ! Ce que je voulais, c'est le faire d'une autre... d'une autre façon!
\end{abstract}

Avec le recul, Edinson explique ce dégoût et plus généralement son manque d'intérêt à poursuivre la formation comme une conséquence directe de l'influence de l'institution catholique.

2. Portela Guarín explique qu'on ne décide pas volontairement de devenir thê 'wala (2002:85-87). C'est un fait qui s'impose par le biais d'une « vision » (généralement du duende, le lutin de la forêt, l'un des trois esprits sur lequel repose la cosmovision nasa). Lors du premier entretien, Edinson a mentionné trois modes de révélation: par une vision en état d'éveil; par les signes ressentis; par une vision dans les rêves.

3. Action de mâcher la coca, en feuille pilée ou en poudre, mélangée à une chaux (cendres, pierres ou coquillages pillés) qui a pour effet de libérer l'alcaloïde de la plante. 
Dans l'imaginaire autochtone, Tierradentro est encore aujourd'hui considéré comme « le cœur de la culture nasa », notamment pour la force du pouvoir spirituel reconnu aux thê'wala de la zone (Rappaport 2008:53,217). Mais en dépit de cet imaginaire, Tierradentro est aussi une place forte du courant conservateur de l'Église catholique dont l'assise s'est construite depuis les premiers temps de la colonisation: dès 1613, des missions parcouraient le territoire pour évangéliser les populations dont la farouche résistance avait mis en échec la conquête militaire. Le colonisateur espagnol avait alors dû repenser sa stratégie d'implantation territoriale en engageant une conquête spirituelle.

Plus récemment, dans la continuité du concordat signé entre l'État et l'Église en 1887, Tierradentro était déclarée « terre de mission » en 1903, puis préfecture apostolique en 1921. L'éducation des indiens revenait dès lors aux religieux et ce, jusqu'en 2004, date à laquelle le Conseil Régional Indigène du Cauca (CRIC) récupère la gestion des écoles (Rappaport 2008:217; Baronnet et Mazars 2010:141-146). Dans ce contexte historique, les thê'wala, considérés comme des concurrents directs du clergé dans le domaine du spirituel, n'ont jamais cessé d'être associés à la figure du diable; leur pratique fut condamnée. Ils ont dû se cacher et pratiquer discrètement les rituels et les consultations. De ce fait, la transmission du savoir a été profondément limitée et transformée à mesure que l'emprise de l'Église catholique grandissait et s'imposait dans la région.

Cette mise à l'écart des autorités autochtones reflète l'état d'une lutte dans laquelle est en jeu " l'imposition de la manière légitime de percevoir » l'ordre du monde (Bourdieu 1980:244), le fait de connaître, de re-connaître, de se référer à un certain nombre de catégories cognitives (Bourdieu 2012:260-261) et de représentations qui expliquent les relations sociales et la place de chacun dans cet ordre. Plus généralement, une catégorie générique s'est imposée pour désigner les populations locales dont les territoires sont à dominer. Ainsi, en paraphrasant Jean-Paul Sartre (1954:75), on pourra dire que c'est le colonisateur qui a fait l'Indigène ${ }^{4}$. Un ensemble de caractéristiques négatives sur le plan moral sont assimilées à cette catégorie, qui permet d'identifier ce qui doit être changé. Ce sont les stigmates de l'indigéneité.

L'éducation donnée par les religieux utilise et reproduit ces catégories pour progressivement faire abandonner les caractéristiques définies comme négatives au profit de ce qui est énoncé comme civilisé, faisant ainsi jouer le " pouvoir structurant des mots » (Bourdieu 1982:150) pour transformer la pratique des enfants. Comme nous allons le voir, cette éducation entre en concurrence avec la socialisation primaire qu'ont connu les enfants nasa dans le cadre familial. Et la confrontation de ces deux formes de socialisation permet ici de comprendre que puissent cohabiter en eux un sentiment de fierté et un sentiment de dégoût pour certains aspects de leur origine sociale.

\section{Désapprendre que l'on est « indigène "}

Comme la plupart des enfants de cette région, Edinson apprend à lire et à écrire dans une école dirigée par des sœurs. Mais il n’y apprend pas seulement cela. On lui intime

4. Ou l'Indio, dans l'usage espagnol courant qui en est alors fait. 
aussi de se défaire d'une partie de son héritage familial, en premier lieu de sa langue maternelle et de son vêtement habituel.

Toutes les classes étaient avec les sœurs, et... dans la salle, nous ne pouvions pas parler dans notre langue. Nous étions punis. Moi, personnellement, je ne pouvais pas entrer avec mon poncho, je ne pouvais pas entrer parce que, ben... c'était interdit. Si bien qu'[elles] m'ont obligé à mettre ces vêtements [...] C'était un poncho noir, que ma grand-mère m'avait offert, et j'étais toujours avec ce petit poncho, et ils m'avaient collé 'Poncho Noir' comme surnom. Et ça m'a donné tant de peine, ben, de jeter ce poncho! Et de ne plus continuer avec ce poncho! Nous avons été rejetés ainsi! Si nous parlions dans notre langue, nous étions punis! [Elles] nous faisaient agenouiller sur le sol, en premier à jeter du maïs ou du sable, et [elles] nous faisaient nous agenouiller, trois, quatre, jusqu'à cinq heures, mains en l'air. Seulement pour avoir parlé! Seulement pour avoir été avec le poncho. Si bien qu'il nous a fallu abandonner nos coutumes.

On voit ici que l'enfant subit l'imposition d'une façon d'être par la punition, par l'exercice d'une violence directe, physique qui vise à incorporer une discipline et rappelle en cela l'idée d'une domination exercée par un processus de normation (Foucault 2004 : 59). Mais la violence n'est pas seulement physique, elle est aussi symbolique: on lui demande de jeter l'objet qui lui a valu son surnom, le poncho noir qui, de surcroît, lui avait été offert par sa grand-mère, membre de la famille généralement associée à l'idée de sagesse et de transmission du savoir. Concrètement, c'est d'une partie de lui dont on lui demande de se défaire. Et l'imposition progressive d'une façon d'être distincte de celle apprise dans le contexte familial contribue à rompre la cohérence d'une organisation sociale.

Les années 1970 voient cependant naître dans les communautés un processus organisationnel qui, s'il part d'une contestation de la structure agraire, s'appuie pour se former sur un discours de revalorisation de l'identité ethnique. En 1972, le CRIC est créé suite à l'initiative des cabildos $^{5}$ de communautés du nord et du centre du Cauca. L'organisation doit permettre d'appuyer et de coordonner les actions d'occupation et de récupération de terres. À Tierradentro cependant, la dynamique rencontre des obstacles et les premières récupérations n'ont lieu qu'à partir de 1975. Pourtant, Tierradentro se caractérise par une forte concentration agraire entre les mains d'un nombre réduit de grands propriétaires terriens. L'Église elle-même possède des propriétés.

La famille d'Edinson est une famille de terrajeros, un statut alors très répandu dans les communautés du Cauca. Pour conserver l'usage d'un lopin de terre, le terrajero doit un certain nombre de jours de travail au propriétaire terrien. Cette condition porte en elle des rapports de profonde inégalité sociale qui, pour être justifiés, s'appuient sur les stigmates de l'indigénéité. Les mobilisations indigènes d'alors en réclament l'abandon parallèlement à une demande de répartition des terres. En somme, il s'agit d'une lutte contre une injustice économique et pour la redistribution (Fraser 2011:19) dont le fondement s'appuie sur la dénonciation d'un statut social basé implicitement sur une différenciation ethnique.

5. Le cabildo, figure d'origine coloniale, est l'instance de gouvernement de la communauté. Il comprend généralement un gouverneur (l'autorité la plus importante), un secrétaire, un trésorier et un capitaine. Le resguardo est le territoire collectif et indivisible de la communauté juridiquement déterminé. 
Condition quasi féodale, le terraje implique des relations d'allégeance qui s'inscrivent de surcroît, à Tierradentro, dans un environnement politique où le jeu clientéliste des partis traditionnels domine. Ainsi, grands propriétaires terriens, membres du clergé et notables locaux se partagent le contrôle de la zone. Dans les communautés, le curé et les autres représentants religieux possèdent un pouvoir incontournable et interviennent jusque dans la désignation des membres du cabildo. Le père d'Edinson, gouverneur à partir de 1971, « aidait le curé » explique-t-il; " il ne possédait pas d'autorité ». Quand le CRIC, tentant d'étendre la mobilisation à Tierradentro, invite les gouverneurs à une réunion, son père se tourne en premier vers le curé pour lui demander conseil. « Ce sont des communistes !», aurait dit le religieux, condamnant tout rapprochement avec le processus naissant qui, toujours selon lui, " n'a rien avoir avec le cabildo ». Puis, un peu plus tard, le père d'Edinson est directement menacé d'emprisonnement s'il s'aventure dans les récupérations.

Dans ce contexte familial et éducatif, il peut sembler étonnant, voire paradoxal, qu'Edinson soit aujourd'hui parmi les promoteurs d'une médecine dite traditionnelle en ayant contribué par exemple, comme nous le verrons un peu plus loin, à la réactivation du rituel du Saakhelu. Edinson a été soumis à l'incorporation des stigmates de l'indigénéité. Il a par ailleurs grandi dans un environnement familial qui respecte l'allégeance à un ordre social structuré autour d'une organisation hiérarchisée de la distribution des pouvoirs et d'une division ethnicisée du travail. En somme, il n'y a pas de prédisposition à la contestation des conditions objectives de la domination.

Si l'on reformule ce paradoxe apparent en l'élargissant, on peut se demander comment surgit un discours propre, un discours qui valorise l'identité ethnique alors que l'indigénéité a été pendant si longtemps assimilée à des valeurs extrêmement négatives. Quelles sont les conditions de l'émergence de la subjectivité collective des dominés, de leur agency ou capacité en tant que subalternes à produire une parole autonome qui, de surcroît, conteste la parole dominante, en somme, à vivre une " subjectivité rebelle » (Negt 2007) ? La problématique n'est pas nouvelle, de nombreux auteurs l'ont abordée-parmi lesquels Bourdieu (notamment 1977 : 2-5), Foucault (2001 : 1041-1062) ou encore Spivak (1988) - et nous ne tenterons pas d'apporter ici une réponse globale. La trajectoire d'Edinson offre cependant l'occasion d'y réfléchir empiriquement. Pour ce faire, nous nous appuyons sur l'idée que les habitus, résultat de trajectoires singulières, sont aussi soumis à des changements réguliers, les dispositions acquises étant réinterprétées et restructurées au gré des expériences et situations nouvelles vécues par les agents, sans être pour autant radicalement modifiées (Bourdieu 2003:231). Ainsi, l'habitus d'un agent n'est pas forcément uniforme, il peut être composé de différentes " couches », être clivé en fonction du degré d'hétérogénéité des situations sociales dans lesquelles il a été et est amené à évoluer (Bourdieu 2003 : 95). La pratique qui en résulte peut exprimer les tensions existantes produites par la cohabitation de ces expériences hétérogènes, voire contraires (Pudal 1989:129). Dans un tel contexte, l'habitus se compose de dispositions plurielles dont la mise en action s'adapte à « la logique de la situation vécue » (Lahire 2001 : 87).

À ce point de la vie d'Edinson, on retrouve cette variété de situations vécues et d'apprentissages incorporés, faisant cohabiter la fierté de se savoir choisi pour devenir " grand sage », un certain type de connaissance culturelle et d'interprétation du monde social par l'héritage familial, avec le dégoût pour ce qu'implique la pratique de thê 'wala, 
résultat d'une éducation l'incitant à se débarrasser des stigmates de l'indigénéité. Edinson est à une position « de frontière », selon l'expression de Rappaport (2008: 7-38), entre le « dedans » du monde nasa et son « dehors ». Cependant, l'effet du déracinement qui le place à cette limite, voire au dehors, se dilue à un moment donné face à l'obligation de faire un " choix » que des événements et des rencontres déterminent et qui lui intiment progressivement de s'identifier à son groupe social d'origine, l'univers nasa.

\section{Réapprendre que l'on est « indigène " pour apprendre à être " autochtone"}

Des événements viennent en effet perturber ce qui conduisait Edinson à un oubli progressif de toute une façon de vivre et de faire. L'événement constitue « une rupture non dans la réalité, mais, [...] dans l'intelligibilité » (Bensa et Fassin 2006:188), c'està-dire qu'il offre un espace où penser le fait social hors du déroulement de la routine des dispositions acquises en rompant le cycle de l'interprétation que ces dispositions et que les catégories cognitives qui lui sont associées impliquent habituellement.

Tout d'abord, même si sa famille reste éloignée des premières réunions convoquées par le CRIC et des premières récupérations qui ont lieu à Tierradentro à partir de 1975, Edinson grandit dans un univers où l'on évoque couramment les mobilisations autochtones. Au terme de sa scolarité, il devient terrajero et, à partir de 1980, participe aux actions de récupération. « Nous étions déjà terrajeros, cela était comme cela ... on devait se rattacher... à la récupération ». Car l'existence du CRIC a ouvert un nouveau domaine des possibles: il est dorénavant pensable de contester le terraje et la concentration des terres, bases de l'organisation sociale de la structure agraire locale.

Les mobilisations autochtones sont aussi le cadre de la contestation d'une restructuration programmée autour de la réforme agraire adoptée en 1961 et par laquelle la répartition des terres devait s'opérer selon un mode individualisé et privatisé. En s'organisant autour de l'occupation collective et de la répartition indivisible des terres, les mobilisations autochtones défendent des valeurs et des principes qui entrent en contradiction avec l'esprit d'une réforme agraire visant à intégrer au développement capitaliste l'espace des campagnes. De même, en contestant le terraje, on conteste l'existence des stigmates de l'indigénéité: l'acte de récupérer permet de dépasser la condition de terrajero, d'indigène assujetti. Il s'agit d'une prise de conscience de conditions objectives communes, mais aussi du caractère relationnel des stigmates de l'indigénéité, de telle façon que peut s'opérer un renversement du stigmate. De façon plus générale, ces actions permettent de retrouver un devenir commun, de se penser en tant que collectif, en tant que communauté (Espinosa 1996), de se redécouvrir indigène. En somme, il y a une reconfiguration des attentes morales et la progressive production d'une économie morale (Fassin D. 2009: 1257) contestant à la fois celle passée et celle à venir.

Depuis le début des récupérations, les participants doivent faire face à une répression à la fois officielle et officieuse qui les pousse progressivement à trouver les moyens d'une autodéfense permettant d'assurer leur survie. La répression venant de l'État est au plus fort entre 1979 et 1981 et des dirigeants sont arrêtés, emprisonnés, torturés. La répression officieuse se traduit quant à elle par l'assassinat de nombreux leaders dont Edinson n'a de cesse de se remémorer la liste. 
$\mathrm{Au}$ cours des années 1970, des groupes d'autodéfense se forment dans les communautés du nord du Cauca et à Tierradentro pour soutenir et accompagner les récupérations de terres et pour défendre les figures du mouvement. Ces expériences se consolident après différentes tentatives et avec l'appui d'une guérilla, le M196 (Peñaranda 2011 :228-229). Un groupe armé s'unifie sur le Cauca, la « garde mobile », mais la perspective est encore celle d'une autodéfense. Edinson rejoint cette garde à dixsept ans, vers 1981. Elle est pour l'essentiel composée de jeunes issus des communautés et encadrés par des leaders, autochtones ou non, ayant contribué à la consolidation du mouvement et du CRIC (Peñaranda 2011 :229). Début 1985, la " garde mobile » prend une nouvelle dimension en devenant une organisation armée, le Commando Quintín Lame $^{7}$ qui se définit comme " porte-parole armé » des communautés (Peñaranda 2011: 246) tout en se positionnant en acteur autochtone à part entière face aux autres belligérants du conflit armé. Edinson participe à différentes opérations dont l'une, avec le « Bataillon America », se déroule sur neuf mois en 1986. Il est l'un des neuf rescapés des quarante-cinq du Quintín Lame ayant participé à cette initiative du M19 ${ }^{8}$. Il y a gagné une expérience nouvelle et devient chef de la commission de Tierradentro. Mais sa motivation n'est plus la même: si le Quintín Lame sert à appuyer les récupérations, il entraîne son lot de morts et bien trop de douleurs dans leurs familles, explique-t-il. Quelque temps après, vers le début de 1987, Edinson négocie sa sortie de la guérilla suite à une opération difficile.

Tout au long de ce séjour dans le groupe armé, il continue de ressentir les « signes » mais il ne sait toujours pas les interpréter. Les thê'wala des communautés traversées interviennent fréquemment auprès des combattants, en leur faisant suivre des rituels de protection où il s'agit de préparer spirituellement et d'harmoniser les énergies, de nettoyer, voire de « donner du pouvoir » (Peñaranda 2011 :279-282). L'intervention des thê'wala n'est pas une pratique nouvelle puisque dès les premières récupérations de terre, ils étaient consultés pour identifier les combattants dont la participation aux opérations était risquée, ou pour se protéger de la réaction de « l'ennemi » (voir Espinosa 1996: 101-102).

Son passage par le mouvement armé ne contribue donc pas à le faire revenir vers son devenir de thê'wala. Par contre, Edinson participe à des " écoles de formation » (Peñaranda 2011:282-286). Il y reçoit une préparation militaire mais aussi politique. On acquiert dans ces espaces de socialisation une connaissance politique générale et des savoirs spécifiques sur l'identité autochtone (Peñaranda 2011 :256-257). Des discussions sont organisées sur la législation qui touche les populations indiennes, sur les raisons du surgissement du mouvement autochtone, sur des questions d'organisation politique interne comme le fonctionnement des resguardos et des cabildos en relation avec la

6. Le M19 est créé après les élections de 1970 suite à l'échec d'un candidat alternatif aux deux partis politiques traditionnels (conservateurs et libéraux).

7. La guérilla prend son nom du dirigeant nasa du début du siècle, Manuel Quintín Lame, qui avait mené des actions armées en défense de la terre et contre le statut de terrajero, tentant entre 1910 et 1920 d'organiser les communautés.

8. Opération sous commandement du M19 réunissant des combattants du Mouvement Révolutionnaire Tupac Amarú du Pérou, du groupe Alvaro Vive ¡Carajo! d’Équateur et du Mouvement Armé Quintín Lame. 
communauté. Bref, c'est aussi une partie de ce qui alimente le discours autochtone qui est appris et discuté lors de ces formations. Ces écoles contribuent à constituer un groupe de futurs cadres communautaires (Peñaranda 2011 :286), des « intellectuels organiques » au sens défini par Antonio Gramsci (1978: 109) qui, par la suite, diffusent le discours et contribuent à le rendre homogène. Ils s'inscrivent dans la constitution d'un espace public alternatif au dominant, c'est-à-dire un contre-public (Fraser 2011:126128) où se forme une opinion qui ne subit pas les effets généraux d'exclusion propres au public « officiel » et où sont énoncés des attentes et des demandes spécifiques.

Pour Edinson, le passage par le Quintín Lame est concrètement l'espace social où il prend conscience et apprend à se revendiquer autochtone, à faire valoir cette reconnaissance et ce, dans un mouvement de retournement des stigmates de l'indigéneité. D'ailleurs, il l'exprime explicitement de cette façon quand il aborde le moment qui signe la naissance de la guérilla : «La première [action] qui a été réalisée, c'était à Santander de Quilichao. Pour démontrer qu'il existait un groupe indianiste... un groupe d'indigènes... mais armés ! ${ }^{9} \gg$ Il signifie ici qu'ils se pensaient comme des indiens, des autochtones car ils étaient des indigènes armés, c'est-à-dire à même de contester la position de dominé que le statut d'indigène impliquait jusqu'alors. Ainsi, au-delà du seul besoin de récupération de terres, il s'agit aussi d'une lutte pour se faire reconnaître.

Par cette métamorphose dans le domaine des représentations et par cette participation à un contre-public, il devient possible de dépasser l'état de mutisme auquel sa condition sociale le condamnait jusqu'à peu, et ainsi de parler, c'est-à-dire de contribuer à produire sa propre représentation. La prise de conscience s'inscrit ici dans le collectif, fruit de rencontres variées, autochtones ou non. Le domaine des possibles est élargi et permet de puiser dans cette couche de l'habitus où les dispositions incorporées dans le cadre familial et communautaire ressurgissent tout en s'actualisant. Ainsi, la « magie performative du social » (Bourdieu 1980:96) peut-elle s'opérer.

\section{Apprendre à devenir thế'wala}

Au sortir de la guérilla, il n'est pas facile pour le jeune homme de revenir à la vie civile. Les négociations de paix entre le Quintín Lame et le gouvernement ne s'ouvrent qu'à partir de juin 1990. Edinson se cache donc un temps du côté de Belalcázar, vivant cette période dans la peur de faire de mauvaises rencontres. Son retour finalement négocié, il revient dans la communauté. Rapidement, il y occupe des responsabilités politiques en devenant secrétaire du cabildo à partir de 1989, puis à partir de 1990 quatre fois gouverneur et deux fois capitaine. Au sein de la communauté, son passage par la guérilla ne semble pas lui porter préjudice. Au contraire, il lui apporte une certaine légitimité. Formé au discours sur une identité autochtone, à la défense d'un mode d'organisation autonome et sachant mobiliser un répertoire d'actions spécifique, il remplit son rôle d'intellectuel organique du mouvement autochtone, ayant dépassé une situation « de frontière »- entre un dedans, celui du monde autochtone, et un dehors,

9. Edinson dit «(...) un grupo indigenista ... un grupo de indios ... ipero armados!». Je traduis ici indígena et indio selon l'usage social qui en est fait et non selon l'usage courant s'appuyant sur la correspondance étymologique des termes : indígena est ici associé au terme d'Indien ou d'autochtone, plutôt valorisé en français, et indio à celui d'indigène, car « indio » a été utilisé par les colons espagnols de la même façon que les colons francophones ont utilisés le terme d'indigène, c'est-à-dire de façon péjorative. 
celui de la société dominante (Rappaport 2008:43-74) véhiculé entres autres par les apprentissages et désapprentissage de l'école primaire - pour s'engager pleinement en faveur de son groupe social d'origine. En 1990 d'ailleurs, une récupération de terres est organisée à Santa Rosa alors qu'il occupe pour la première fois le poste de gouverneur.

C'était pour qu'ils nous donnent rapidement [la terre]. Nous étions en création de resguardo. Et on a d̂u y mettre beaucoup de médecine. Les précédents [dirigeants] avaient fait de même. [...] Pour pouvoir... on le faisait... pour se libérer des ennemis.

Sa position de dirigeant communautaire le conduit à rencontrer fréquemment des thê'wala. «J'étais toujours à côté... des thê'wala, et petit à petit... j'ai commencé à acquérir des connaissances... j'étais toujours en train de demander, "regarde, à moi, cela me fait telle chose"». Il accompagne aussi un temps l'un de ses oncles dans ses consultations. Mais ce sont surtout deux nouveaux événements qui le conduisent à s'engager plus fermement dans la voie de l'apprentissage.

Edinson connaît une expérience personnelle marquante qui a valeur de confirmation et d'injonction à poursuivre sérieusement sa formation. Le thê'wala de Tumbichucué ne cesse de lui rappeler qu'il doit cultiver ses plantes:

"Va faire tes recherches sur les plantes! Va planter! Pour qu'également ton esprit aille... prenne du pouvoir en s'incarnant dans ton corps!' Mais je n'y ai pas porté attention, jusqu'à ce que finalement, je me trouve... par là-bas, dans ce canyon, près du rocher, par ici, je crois, sur ce rocher, et finalement, ici sur ce bord, j'ai dit "non! Sapristi! Quelque chose est en train de m'arriver!" Et j'ai envoyé ma femme voir une médecin, qui vit par là-bas, qui est bien âgée. Pour la consulter et pour m'envoyer un remède. Pour... me faire un bain, et elle apporte la nouvelle et me dit qu'elle m'avait dit "L'homme a déjà ses pouvoirs et il ne prête pas attention à l'esprit qu'il a". C'est-à-dire le ksxa'w. "Il doit lui porter attention!" C'est-à-dire que je devais obéir à ce qu'ils m'avaient dit. Et donc j'ai tellement pris peur qu'à partir de là, j'ai commencé à cueillir des plantes et à enquêter de nouveau, jusqu'à maintenant! ».

La manifestation a lieu près d'une source d'eau et de rochers, c'est-à-dire là « où résident les esprits tutélaires de la nature » (Dexler 2007:145), dans un environnement qui porte une symbolique particulière dans l'imaginaire nasa (Portela Guarín 2002:33-34). Ce moment est décisif car il constitue une rupture par laquelle se révèle pleinement la dangerosité du pouvoir. Edinson finit par se soumettre aux injonctions des autres thê'wala car ne pas suivre cet apprentissage, refuser «l'alliance avec le monde-autre » (Perrin 1997 :91) se révèlerait aussi dangereux que l'épisode qu'il vient de vivre.

Le contexte social de son apprentissage lui est fourni à la suite d'un autre événement qui touche cette fois-ci le collectif. L'épisode, nouvelle rupture dans l'intelligibilité, nous intéresse ici plus particulièrement, car il illustre une mise en pratique du discours qui va conduire au renforcement d'une autonomie politique et territoriale.

Le 6 juin 1994, un séisme occasionne d'immenses coulées de boue dont la plus meurtrière se déverse dans la vallée du fleuve Páez. On compte plus de 300 morts, 500 disparus et les dégâts dans les communautés de Tierradentro sont considérables. La catastrophe donne lieu à une interprétation de la part des thê'wala et des « anciens » de la région (Rappaport 2008 :213). Ils y voient un « rappel à l'ordre de la Nature », face 
aux usages néfastes de la terre, par exemple la pratique du brûlis, le développement des cultures illicites telles le pavot, ou encore l'absence d'actions de purification et d' « harmonisation » des énergies que les thê'wala d'antan pratiquaient sur le territoire. La catastrophe, ce qui aurait pu être interprété comme une fatalité, un fait du hasard aux conséquences désastreuses, est repensée comme le résultat d'un déséquilibre qui a provoqué une réaction depuis le monde-autre. Cette interprétation mobilise l'idée de " rapports sociaux » entre l'humain et le non-humain. Elle convertit « l'aléa en certitude » (Perrin 1997:92), ce qui, de ce fait, implique d'agir. Partant du constat que Tierradentro n'est plus « soignée » par la pratique de rituels et que cette terre est abandonnée de toute médiation avec le monde-autre, il est décidé dans le cadre de l'organisation zonale affiliée au CRIC, l'Association Juan Tama, de mener une longue enquête auprès des plus anciens pour réapprendre à effectuer de grands rituels. Edinson s'implique pendant six ans ${ }^{10}$. Un groupe consulte les personnes les plus âgées, les thê'wala de Tierradentro mais aussi ceux de la zone nord et leurs homologues parmi d'autres peuples du Cauca.

Nous croyions que les meilleurs [thê'wala] étaient ici, à Tierradentro. Mais, eh bien... il nous a fallu échanger avec d'autres... médecins du nord du Cauca, ainsi que ceux de la zone est. De plusieurs zones. Et... donc c'était une rencontre régionale ${ }^{11}$, c'était avec tous ceux du Cauca.

En 2000, au terme de l'enquête, le rituel du Saakhelu ${ }^{12}$ est de nouveau pratiqué à Tierradentro plus de 130 ans après sa dernière réalisation selon les informations recueillies. Près de deux mille personnes y participent et depuis, chaque année, au solstice d'hiver, le rituel se tient dans cette région tandis qu'un autre est réalisé au solstice d'été dans la zone nord. Trois autres rituels sont « redécouverts » et régulièrement pratiqués : le rituel pour raviver les bâtons de commandement ${ }^{13}$, le rituel pour éteindre le feu ${ }^{14}$, et le rituel du Çhapuc ${ }^{15}$.

On voit ici que la réponse à la crise a été de se tourner vers la tradition. On la mobilise, quitte à adapter le discours à l'absence de sa pratique pour produire un sens et une action qui appuient l'idée d'une identité commune et d'un devenir commun. Le

10. Edinson a aussi occupé divers postes de responsabilité dans cette organisation zonale (trésorier, contrôleur, vice-président).

11. Voir Rappaport (2008:224-225) à propos des réunions et ateliers de travail entre thê 'wala à la suite de la catastrophe de 1994.

12. "Rituel de paiement à la terre mère pour "sacraliser" les graines et garantir une santé intégrale individuelle, familiale et communautaire » (CRIC 2007:49).

13. Rituel du « refrescamiento de varas »: ce sont les bâtons de commandement que les autorités locales conservent à leur proximité au quotidien pour signifier leur pouvoir. Le bâton doit aussi contribuer à leur rappeler constamment leurs responsabilités. À la fin du mandat, il est transmis au successeur. Selon la définition donnée par le programme de santé, il s'agit d'un « rituel qui oriente les actions des autorités et prévient la rupture de l'équilibre à cause de problèmes d'ordre interne, rupture qui génère un malaise physique, spirituel et collectif (la maladie selon la conception nasa)» (CRIC 2007:49).

14. «Rituel de prévention des maladies, des maux physiques, culturels et spirituels, qui contribue à maintenir des conditions d'harmonie et de protection du territoire et de la communauté » (CRIC 2007:49).

15. « Rituel de paiement aux esprits et au territoire » (CRIC 2007:49). 
pragmatisme des enquêteurs, qui les pousse à se tourner vers les pratiques de peuples voisins et donc à produire une forme de syncrétisme, est l'exemple concret que ce n'est pas tant l'authenticité qui importe que le fait de produire du sens par lequel on se donne une unité à partir de laquelle il devient possible d'agir. Il s'agit de mobiliser le groupe, de lui faire [re]-connaître ce qui lui donne sa cohérence, de le faire se reconnaître et de le faire reconnaître à l'extérieur. Bref, ce processus d'ethnogenèse, qui s'inscrit dans un contexte général de résistance (Boccara 1999:458) et par lequel des rites sont réactualisés, contribue à redéfinir les termes des rapports sociaux et des identifications.

\section{"Chamanisme» et construction del'autonomie territoriale et politique}

Rappaport a noté l'importance des thê'wala dans la production d'une histoire orale des Nasa (2008:216), qui a été progressivement écrite notamment grâce à l'enquête d'un petit groupe d'activistes locaux entreprise dès la fin des années 1980 (2008:221225). Soulignant leur sens de l'interprétation des contextes politiques, l'enquête révèle que les « grands sages » ont favorisé au cours du temps une histoire négligeant le mythe des origines (Rappaport 2008:224), où deux entités Uma et Tay créent le monde, pour préférer conter la trajectoire mythifiée d'une figure indienne historique du début du $18^{\text {ème }}$ siècle, Juan Tama, lequel avait réussi à imposer face aux Espagnols la catégorie du resguardo et ainsi à sauvegarder pour partie une autonomie territoriale (Rappaport 2000: 89-110). C'est que les thê'wala ont un rapport particulier avec le territoire. Ils rappellent la signification de certains espaces. Par leur méditation, par les signes qu'ils reçoivent et par la connaissance qu'on leur transmet, ils sont à même de désigner les lieux sauvages et dangereux, incultivables, mais aussi sacrés, où l'on entre en contact avec le monde-autre (Portela Guarín 2002:107). Ils classifient l'espace en associant certains endroits à des caractéristiques, en les nommant, en les organisant et en les expliquant selon une logique symbolique. Ils participent de ce fait à la redéfinition du territoire, comme en témoigne l'interprétation de la catastrophe de 1994, et occupent une place non négligeable dans la lutte pour l'imposition des catégories légitimes d'interprétation de l'ordre social. Par ailleurs, comme l'illustre leur action auprès des récupérateurs de terres et des combattants du Quintín Lame, leur intervention sur le territoire ne se limite pas au seul domaine du symbolique. Ils y agissent directement au travers des rituels, ces « stratégies de santé territoriale » (Drexler 2007 : 156) effectuées dans les lieux sacrés. Ils participent ainsi à une dynamique plus large de reconquête des territoires des communautés.

L'après-catastrophe fournit de fait le contexte à un investissement du CRIC à Tierradentro. L'aide humanitaire destinée aux sinistrés est pour partie gérée par des ONG qui se tournent vers les militants locaux pour mobiliser leur savoir-faire, tandis que la Corporation Nasa Kiwe à laquelle est initialement associé le CRIC doit gérer la relocalisation des communautés déplacées par la catastrophe et planifier des plans de développement. Mais surtout, la nécessité d'un appui sanitaire fournit le contexte à la création en 1997 de l'AIC, organisme d'administration de la sécurité sociale directement géré et contrôlé par le CRIC. Les déplacés de la catastrophe sont les cinq mille premiers affiliés de l'AIC. L'organisme s'insérant dans un système de santé privatisé, les autorités des cabildos ont conditionné sa création à la défense d'un régime spécial autochtone devant appuyer une approche intégrale et le développement d'une médecine dite traditionnelle (Mazars 2012:261-262; Mazars 2013:478-479). 
Les centres de soins créés localement par les organisations zonales et financés par l'AIC concentrent donc leur intervention (aussi pour des raisons matérielles) sur la promotion et la prévention, ainsi que sur l'appui des praticiens locaux. Concrètement, il s'agit de prévenir les maladies par la diffusion de mesures basiques d'hygiène, par l'apprentissage de l'usage de plantes médicinales, par la défense d'une souveraineté alimentaire en aidant à la culture de produits variés, ou encore d'aider à la revalorisation des rituels en accompagnant les thê 'wala, tandis que les soins allopathiques sont fournis par les hôpitaux municipaux et régionaux (Baronnet et Mazars 2010:168-171). Entre 2001 et 2005, Edinson est " coordinateur de médecine traditionnelle » pour le centre de soins d'Inzá, Nasa Çxha Çxha IPSI. Il a à sa charge l'organisation pratique des grands rituels; il est en contact avec les autres thê 'wala de Tierradentro et appuie leurs initiatives de réunion. À partir de 2003, le centre de soins héberge les consultations des « médecins traditionnels ». Mais en 2005, l'expérience est arrêtée, car les autorités des cabildos dénoncent une tendance à la commercialisation contraire à la logique initiale de cette pratique médicale. Si les consultations restaient gratuites, ou du moins compensées par des dons en nature (par exemple de la nourriture préparée) comme cela se fait habituellement dans les communautés, une pratique de vente de plantes et de remèdes médicinaux commençait à se développer. En somme, le contexte appuyait une marchandisation de l'acte de soins, facette du système de santé pourtant dénoncée dans le discours. Cette expérience met en relief les effets de pénétration d'une pratique et l'on peut se demander, comme le propose Espinosa, si cette implication des militants autochtones dans la gestion des ressources, en vertu de l'application d'une politique publique, ne transforme pas ces militants en « quasi-agents de l'État» (1997: 128). Plus précisément, en participant à la mise en place d'un système de santé - c'est-à-dire à l'application d'un modèle dominant et de sa logique matérielle et symbolique -, n'ontils pas perdu les acquis du contre-public qui se fragilise avec l'expérience de pratiques contradictoires au discours développé en son sein ?

Cette problématique touche à différentes dimensions. Une interprétation serait de dire qu'ils « se battent à l'intérieur de l'État» (Laurent 2012:157). Le cas de l'AIC illustre effectivement une situation de «lutte agonistique » (Mouffe 2010: 18-24; Mazars 2012:264; Mazars 2013:17-22): les employés de l'organisme autochtone appliquent un système de santé tout en le contestant et en se confrontant aux fonctionnaires nationaux pour obtenir la reconnaissance de leur spécificité autochtone et celle d'un régime autochtone spécial. De notre point de vue, cette participation nous semble surtout être le moyen trouvé pour construire une autonomie forte (Gros 2010:180182), gouverner et administrer les territoires, en se positionnant en intermédiaire entre les communautés et l'institution étatique. Pour ce faire, le discours d'une défense de la «médecine traditionnelle » et de la cosmovision légitime l'intervention dans les communautés et conduit à l'appui des autorités locales. En retour, la gestion de la santé rend possible une présence directe dans les communautés et la réappropriation d'un biopouvoir que permet la constitution d'un savoir sur une population grâce, entre autres, aux connaissances statistiques générées par l'affiliation et les rapports de santé. Fin novembre 2009, les chiffres de l'affiliation rendaient compte d'une confiance certaine acquise par l'AIC auprès des communautés, car selon la loi, le choix de l'organisme de sécurité sociale est décidé collectivement et la décision ratifiée (par exemple en assemblée communautaire) et rapportée par l'autorité locale. L'AIC affiliait 246308 
personnes sur toute la Colombie ${ }^{16}$ et gérait un budget d'environ 23 millions d'euros. À Tierradentro, elle avait pour la même période la responsabilité sanitaire de la quasitotalité des autochtones connus dans le système de santé de cette région ${ }^{17}$.

L'AIC s'appuie donc sur la revendication et la mise en pratique d'une médecine autre, labélisée autochtone, pour justifier son intervention. Le label autochtone est aussi mobilisé pour désigner une situation sanitaire fragilisée (isolement géographique, mauvaises conditions épidémiologiques en raison d'une pauvreté pérenne, etc.) dont la résorption n'est possible qu'avec l'obtention de ressources économiques supplémentaires. En somme, le conflit qui oppose ses agents aux représentants de l'État s'inscrit dans les termes d'une lutte pour la reconnaissance dont le fondement se base sur la dénonciation d'une inégalité socio-économique. Depuis les récupérations de terres des années 1970 et 1980, les termes du discours autochtone se sont transformés, mais aujourd'hui comme hier persiste la dénonciation d'un déni d'égalité socio-économique et d'un déni de reconnaissance. S'il y a une mutation du discours vers la promotion accrue de la reconnaissance de l'identité autochtone, cela reflète avant tout une adaptation pragmatique des médiateurs autochtones qui, pour se faire entendre, recourent au thème de la reconnaissance. Elle est en effet devenue la forme paradigmatique, et donc dominante, au travers de laquelle sont interprétés le conflit politique et les moyens de sa résolution (Fraser 2011:13). Mais cela ne représente que l'état actuel d'une lutte dont l'enjeu est l'interprétation des raisons du conflit politique et social. Sur le fond, reconnaissance et redistribution restent deux luttes intrinsèquement liées et se renforçant dialectiquement (Fassin et Fassin 2009: 262-263 ; Fraser $2011: 30)$.

En 2008, Edinson explique avoir encore énormément de choses à apprendre. Peu de personnes de la communauté le consultent, le considérant comme «trop jeune ». Il lui semble même être mieux apprécié à l'extérieur. Il s'est aussi formé auprès des « sages » d'autres peuples. Ainsi, en 2000, il est resté quelques temps l'apprenti d'un taita ${ }^{18}$ kamsá ${ }^{19}$ avec lequel il s'est initié au yajé qui, comme les champignons hallucinogènes, lui permet d'amplifier la perception des signes.

Fin 2011, Edinson semble beaucoup plus sûr de lui. Il soigne plus régulièrement dans la communauté et il forme lui aussi de futurs thê'wala. Cinq apprentis le suivent régulièrement dont deux ne sont pas de la région. Tout comme il a enrichi sa pratique de techniques apprises chez d'autres peuples, Edinson diffuse ses connaissances au-delà de Tierradentro. C'est que la pratique chamanique, à l'image de la vie des gens et à l'image de la construction du discours autochtone, est en perpétuel mouvement.

16. Dont 197476 personnes dans le Cauca, parmi lesquels 174307 sont identifiées comme autochtones dans le système de santé (source: informations compilées par l'auteure à partir de données du ministère de la Protection Sociale colombien, 2010).

17. Soit 30794 personnes sur un total de 31968 (source: ministère de la Protection Sociale colombien, 2010).

18. Taita: homologue du thê'wala chez différents peuples de la région.

19. Peuple du Putumayo, un département situé au sud du Cauca et frontalier avec l'Équateur. 


\section{Références citées}

Baronnet, Bruno et Nadège Mazars, 2010. « Los pueblos indígenas de Colombia frente a los servicios públicos de salud y educación : las experiencias de gestión propia de la política social », in Jean Foyer et Christian Gros (éds), ¿Desarrollo con identidad?, 121-184. Lima: CEMCA, IFEA, FLACSO.

Bensa, Alban et Éric Fassin, 2006. «Les sciences sociales face à l'événement », in Alban Bensa, La Fin de l'exotisme. Essais d'anthropologie critique, 171-195. Toulouse: Anacharsis.

BoccAra, Guillaume, 1999. «Etnogénesis mapuche: resistencia y retructuración entre los indígenas del centro-sur de Chile », The Hispanic American Historical Review, 79 (3): 425-461.

Bourdieu, Pierre, 1977. « Une classe objet », Actes de la recherche en sciences sociales, 17-18:2-5.

-, 1980. Le Sens pratique. Paris: Les éditions de Minuit.

-, 1982. Ce que parler veut dire. L'économie des échanges linguistiques. Paris: Fayard.

-, 2003. Méditations pascaliennes. Paris: Seuil.

-, 2012. Sur L'État. Paris : Raisons d'Agir/ Seuil.

CRIC, 2007. Programa de salud del CRIC. Popayán, archives personnelles.

DREXLER, Josef, 2007. «Las "siembras de agua": La concepción y las prácticas de salud territorial de los nasa (páez) de Tierradentro en Colombia. Otra mirada indígena a la reforestación », Anthropológicas, 18 (1), 137-170.

Espinosa, Myriam A., 1996. Surgimiento y andar territorial del Quintín Lame. Quito: Abya Yala.

-, 1997. « Práctica social y emergencia armada en el Cauca », in María Lucía Sotomayor (éd.), Modernidad, Identidad y Desarrollo, 111-130. Bogota: Instituto Colombiano de Antropología.

FAssin, Didier, 2009. «Les économies morales revisitées », Annales. Histoire, sciences sociales, 6, 64e année: 1237-1266.

FASSIN, Didier et Éric FASSIN (dir.), 2009. De la question sociale à la question raciale ? Représenter la société française. Paris: La Découverte.

Foucault, Michel, 2001. Dits et écrits, tome 2. Paris: Gallimard.

-, 2004. Sécurité, Territoire, Population. Cours au Collège de France 1977-1978. Paris: Éditions Hautes Études, Gallimard, Seuil.

Fraser, Nancy, 2011. Qu'est-ce que la justice sociale? Paris: Editions La Découverte.

Gramsci, Antonio, 1978. Cahiers de prison. Tome 3. Paris : Gallimard.

Gros, Christian, 2010. Nación, Identidad, Violencia : el desafio latinoamericano. Bogota: EPRI, Université Nationale de Colombie CESO, IFEA.

LAHIRE, Bernard, 2001. L'Homme pluriel. Les ressorts de l'action. Paris : Armand Colin-Nathan.

LAURENT, Virginie, 2012. « Dans, contre, avec l'État: mouvement indien et politique(s) en Colombie, vingt ans après », in Christian Gros et David Dumoulin Kervran (éds), Le multiculturalisme " au concret ». Un modèle latino-américain?, 147-158. Paris : Presses Sorbonne Nouvelle.

Mazars, Nadège, 2012. "Santé et Multiculturalisme en Colombie: l'épreuve de la pratique », in Christian Gros et David Dumoulin Kervran (éds), Le multiculturalisme " au concret ». Un modèle latinoaméricain?, 255-266. Paris: Presses Sorbonne Nouvelle.

-, 2013. Les Ruses de la pratique subalterne. La santé gérée par les autochtones en Colombie, un multiculturalisme de domination et/ou d'autonomie? Thèse de sociologie présentée à l'Université Sorbonne Nouvelle - Paris III.

Mouffe, Chantal, 2010. «Politique et agonisme », Rue Descartes, 67 : 18-24.

Negt, Oskar, 2007. L'Espace public oppositionnel. Paris : Payot. 
Peñaranda, Daniel R., 2011. Résistance et reconstruction identitaire dans les Andes colombiennes. Le mouvement armé Quintin Lame. Thèse de sociologie présentée à l'Université Sorbonne Nouvelle Paris III.

Perrin, Michel, 1997. « Chamanes, chamanismes, chamanologues », L'Homme, 37 (142): 89-92.

Portela Guarín, Hugo, 2002. Cultura de la salud Páez: Un saber que perdura, para perdurar. Popayán: Editorial Universidad del Cauca.

Pudal, Bernard, 1989. Prendre parti. Paris : FNSP.

RAPPAPORT, Joanne, 2000. La Política de la memoria. Popayán: Editorial Universidad del Cauca.

-, 2008. Utopías interculturales. Intelectuales públicos, experimentos con la cultura y pluralismo étnico en Colombia. Bogota: Editorial Universidad del Rosario.

SARTre, Jean-Paul, 1954. Réflexions sur la question juive. Paris: Gallimard-Folio.

SpIvaK, Gayatri C., 1988. « Can the Subaltern Speak?», in Carry Nelson et Lawrence Grossberg (éds), Marxism and the Interpretation of Culture, 271-313. Chicago: University of Illinois Press. 